\title{
THESIS ABSTRACT
}

\section{Enzymatic and Cytogenetic Study on Patients with Colon Cancer}

\section{Hzima Mossa Al-Abassi}

A Ph D thesis conducted in Almustansyria University College of Science, 2001, under the supervision of Professor Esmail K Shubber and Professor Hussein H. Khanaka.

Background: Globally cancer is the second leading cause of death. Lung, prostate, colorectal, stomach and liver cancer are the most common types of cancer in men, while breast, colorectal, lung, cervix and thyroid cancer are the most common among women. The cancer burden can also be reduced through early detection of cancer and management of patients who develop cancer. Many cancers have a high chance of cure if diagnosed early and treated adequately. In Iraq, colorectal cancer is the third cancer type.

Aim: This study aiming to determine the predictive value of some variables for the early diagnosis of colorectal cancer and their influence on cancer cells growth.

Methodology: The investigation had been done on the peripheral blood sample which drawn from patients with colon carcinoma by using peripheral blood culture technique. There were three-study groups:

- First group (preoperative group) patient at pre-clinical stage (15 samples male and female).

- Second group (postoperative group) patient after surgical resection of intestinal mucosa and they were under treatment with cytotoxic drug (15 samples male and female).

- Control group. Which included (25 healthy persons male and female).

The cytogenetic study has been done in order to define the damaging effect of the cancer and the anticancer drug in the genetic material of the patient. These damages were manifested through the 
significant reduction in the blastogenic index (BI). Mitotic index and replicative index (RI) in the lymphocyte cells of patient. This accompanied by significant increase in sister chromatid exchange (SCE). In addition to investigate mutation fraction (MF) for two genes (HGPRT, DHFR) by culturing cells in selective medium which contain selective agents (6-TG, MTX) respectively. The result was $26 \%$ of MC which represent the resistant cell to the drug which mentioned abroad spectrum of mutagens and useful genetic marker for DNA-mediated transformation of mammalian cells.

The second part of this study represent the enzymatic study that included three enzymes, HGPRT, DHFR, and ADA.

- (Hypoxanthin-Guanin phosphoribosyl transferase) HGPRT which involve in the synthesis of IMP (inosine mono phosphate) by salvage pathway. This enzyme is responsible for the sensitivity of cells to purine analogues drug such as 6Thioguanine.

- (Dihydrofolate reductase) DHFR which catalyzes the NADPHdependent reduction of dihydrofolate to tetrahydrofolate which is in turn plays a control metabolic role as a carier of onecarbon unit in the biosynthesis of purine and pyrimidine basis so it is play a major role in the DNA synthesis.

- (Adenosine deaminase) ADA, which represents the immune enzyme it is responsible for, catalyzes the deamination of adenosine and deoxyadenosine to inosine and deoxyinosine respectively.

Results: The major finding of this study were as follows:

- A simple and rapid spectrophotometric assay has been developed in order to measure the activity of HGPRT in human erythrocyte lysate and represent an alternative assay instead of radio-chemical assay which is very expensive, timeconsuming rather than it cannot be used in our country because the hard and fast embargo. The result was significantly reduced specific activity in the two studying groups in comparison with control so the finding suggest that there was a relation between the mutant from of this enzyme and the disease (cancer) so this assay may can be use as a simple tool for early detection of the disease especially the sporadic colon carcinoma (SCC). 
- The DHFR has higher specific activity in the second group with respect to control while the first group has differs specific activity of enzyme according to the age in comparison with control group.

- The ADA has reduced specific activity in comparison with control group.

The studies on purine and pyrimidine metabolism have shown how a biochemical imbalance may be responsible for a selective growth advantage for tumor cells and explain their faster proliferation with respect to normal tissue.

Conclusion: HGPRT determination was a simple tool for the early diagnosis of colon cancer. Additionally, the biochemical imbalance may serve as risk factor for tumor cell growth. 